\title{
Transparência do Banco Central: uma análise para o caso brasileiro
}

\author{
HELDER FERREIRA DE MENDONÇA \\ ADRIANA INHUDES*
}

Central Bank transparency: an analysis of the Brazilian case. Nowadays there is a tendency among central banks of increasing transparency in the conduction of the monetary policy. After the adoption of inflation targeting in Brazil there was an increase in the communication of the Central Bank of Brazil with the public. This paper makes a brief review of the recent theoretical and empirical literature concerning this subject. Furthermore, an analysis due to the transparency in the conduction of Brazilian monetary policy on important macroeconomic variables is made. The findings denote that an increase in transparency improves the behavior of several macroeconomic variables.

Key words: transparency, monetary policy, inflation targeting.

JEL classification: E52, E43.

\section{INTRODUÇÃO}

Hoje em dia há uma tendência entre os bancos centrais para aumentar a transparência na condução da política monetária. De acordo com Mishkin (2004) um importante benefício da transparência em um regime de metas de inflação é tornálo mais democrático. Além disso, há um incremento na accountability do formulador de política econômica que contribui para a redução da ocorrência de inconsistência dinâmica por meio de políticas inflacionárias.

A definição mais comum de transparência do banco central é aquela que a relaciona com a ausência de informação assimétrica entre os formuladores de política monetária e outros agentes econômicos. De acordo com Geraats (2002), tal definição de transparência refere-se às informações que os agentes possuem de fato, e não apenas à sua simples divulgação. Assim, caso alguma manipulação de dados seja necessária para extrair informações úteis dos dados disponíveis e o mercado não possua tais recursos, a assimetria de informações persistirá na economia. 
No Brasil, depois da adoção do regime de metas para inflação, ocorreu um incremento na comunicação do banco central com o público o que, por conseguinte, indica um aumento no grau de transparência da política monetária. Não obstante, as possíveis análises do impacto deste incremento sobre os resultados econômicos e as expectativas do setor privado ainda são escassas. Destarte, este artigo faz uma revisão da literatura recente sobre o assunto e faz uma avaliação, para o caso brasileiro, do efeito da transparência na condução da política monetária sobre importantes variáveis macroeconômicas.

Além desta introdução, o artigo encontra-se organizado da seguinte forma: a segunda seção realiza uma revisão teórica sobre a transparência do banco central; a terceira apresenta evidências empíricas encontradas na literatura; a quarta seção faz uma avaliação do impacto do aumento da transparência na condução da política monetária no Brasil; por último, é apresentada a conclusão.

\section{ASPECTOS TEÓRICOS SOBRE TRANSPARÊNCIA DO BANCO CENTRAL}

A análise teórica sobre transparência do banco central deriva do debate regras versus discrição e está presente na literatura como uma tecnologia de comprometimento que induz a autoridade monetária a cumprir os objetivos predeterminados. As principais explicações para adoção de uma política monetária mais transparente são de ordem política. Um aumento na transparência da autoridade monetária reforça a legitimidade democrática de um banco central independente e, de certa forma, o protege de pressões de ordem política. Portanto, há um aumento na accountability do banco central devido ao maior monitoramento das ações da autoridade monetária por parte da população.

De acordo com Geraats (2002), dois efeitos estão relacionados à transparência: o efeito incerteza e o efeito incentivo. O primeiro está relacionado ao fato de que a presença de informação assimétrica gera incerteza para os agentes econômicos que precisam aprender por experiência própria e permite a outros tirarem vantagem da presença de informação privada. O segundo efeito refere-se à tentativa dos agentes econômicos que possuem acesso à informação privada influenciarem o comportamento dos demais por meio da divulgação de informações.

Em geral, os modelos teóricos existentes para a análise dos efeitos da transparência concentram-se na questão da incerteza sobre as preferências do formulador de política monetária, ou a incerteza a respeito de choques sobre a economia. Faust e Svensson (2001) afirmam que um maior grau de transparência sobre as preferências e intenções da autoridade monetária torna a credibilidade e a reputação mais sensíveis à condução da política monetária. Além disso, sob determinadas circunstâncias relacionadas ao tipo de informações divulgadas, induz o banco central a seguir uma política próxima à socialmente ótima. Segundo Walsh (2003), um regime de metas para inflação estrito (voltado exclusivamente para a estabilidade de preços) está em conformidade com uma estrutura que induz o banco central a ser transparente e divulgar suas verdadeiras informações. 
Faust e Svensson (2001) fazem uma diferenciação entre três tipos de regimes: um primeiro de transparência mínima, no qual o objetivo com relação ao emprego e as intenções do banco central não são divulgados; um segundo com elevado grau de transparência, no qual as intenções do responsável pela política podem ser observadas pelo público, e este pode estimar as preferências da autoridade monetária; e um terceiro em que há transparência extrema, ou seja, tanto a meta para o emprego quanto as intenções do banco central podem ser diretamente observadas pela sociedade.

Seguindo a perspectiva dos autores supracitados, no caso de transparência elevada, as expectativas de inflação do mercado e a reputação do banco central tornam-se mais sensíveis às ações da autoridade monetária. Logo, há um aumento no custo de qualquer política que se desvie daquela anunciada e, como conseqüência, impede a manifestação do viés inflacionário. O resultado é que a taxa de inflação e as variabilidades da inflação e do emprego são mais baixas. Portanto, transparência elevada sobre as intenções do banco central é, em geral, benéfica para a sociedade, porém representa um custo para o gestor da política monetária.

No caso de transparência extrema as preferências da autoridade monetária não precisam ser estimadas pelo público, ou seja, as ações do banco central não interferem na sua reputação. Assim, a autoridade monetária ganha liberdade para promover políticas de estabilização frente a choques sem gerar perda de reputação. Todavia, o equilíbrio resultante não é vantajoso, pois implica uma média de inflação e variabilidades de inflação e emprego acima daquelas obtidas pelos outros regimes. Logo, nem sempre um aumento da transparência está ligado a melhores resultados de política e maior credibilidade.

O resultado supradito é similar à diferença entre informação total e transparência total elaborada por Jensen (2002). Em um regime de transparência total, a divulgação de informações referentes à condução da política monetária permite ao mercado inferir as verdadeiras intenções do banco central. Ou seja, as ações da autoridade monetária influenciam as expectativas do mercado e a sua reputação. Por outro lado, em um regime de informação total o público não faz inferência sobre as intenções da autoridade monetária. Logo, as expectativas do mercado não reagem às ações do formulador de política econômica.

Para Jensen (2002) informação total e transparência total diferem de forma marcante no que diz respeito à maneira como os incentivos da autoridade monetária são afetados. Bancos centrais com credibilidade alta deveriam adotar um regime de informação total ao invés de transparência total. A justificativa é que este tipo de regime permite que políticas de estabilização, quando necessárias, sejam implementadas sem a perda de reputação. Por sua vez, bancos centrais com baixa credibilidade deveriam adotar uma política de transparência total a fim de construir sua credibilidade junto ao mercado. Em outras palavras, o regime de transparência total deveria ser transitório e ter como objetivo desenvolver a credibilidade. Depois de alcançado o estágio em que a credibilidade está consolidada o regime a ser adotado deve ser o de informação total.

A literatura sobre transparência destaca a presença de alguns trade-offs. Wal- 
sh (2003) afirma que o peso ótimo a ser dado pelo banco central à meta de inflação (como objetivo de política econômica) e o grau de transparência são determinados considerando aspectos contraditórios. Por um lado, o responsável pela condução da política monetária deve levar em conta a necessidade de evitar pressões do governo que levem à manifestação do viés inflacionário. Por outro lado, a política monetária deve ter alguma discricionariedade para que seja possível responder a choques sobre a economia.

No caso de o banco central ter uma política transparente, ou ainda, se o acesso da autoridade monetária às informações sobre os choques é limitado, maior peso será dado à meta inflacionária. Assim, a necessidade de uma política mais flexível para promover a estabilização é menor. Ademais, a transparência faz com que a condução da política monetária esteja menos sujeita às pressões do governo para a promoção de políticas inflacionárias devido ao monitoramento público (aumento da accountability). Destarte, há a presença de um trade-off entre accountability e estabilização do qual depende o grau de transparência na condução da política monetária. A autoridade monetária é mais transparente quanto maior for a accountability e menor a necessidade de estabilização de choques.

Outro trade-off relacionado à transparência foi identificado por Jensen (2002). $\mathrm{O}$ autor examinou o grau ótimo de transparência do banco central e concluiu que este envolve um trade-off entre ganhos com credibilidade e perdas com flexibilidade. Por um lado, a transparência facilita a formação das expectativas do mercado e a inferência de resultados econômicos futuros. Portanto, qualquer medida adotada pelo banco central influenciará de forma significativa as expectativas do público e os resultados econômicos correntes forçando a autoridade monetária a ser mais cautelosa em suas decisões. Por outro lado, no caso de a política monetária ser dotada de credibilidade, a transparência acaba por impor restrições importantes à ação do banco central diminuindo sua flexibilidade para responder a choques. Portanto, o grau ótimo de transparência deve ser mais elevado quanto maior a presença de pressões políticas e menor a necessidade de promoção de políticas de estabilização.

Em análise recente, de Mendonça e Simão Filho (2007) mostram que um aumento na transparência econômica contribui para uma queda nas taxas de inflação e de juros. Além disso, os autores mencionados verificaram que uma maior variância na transparência do banco central reduz o incentivo para a obtenção das metas de inflação. Logo, uma perda na reputação devido ao aumento na incerteza sobre a transparência do banco central prejudica o controle do viés inflacionário na condução da política monetária.

\section{EVIDÊNCIAS EMPÍRICAS NA LITERATURA}

Chortareas et al (2001) construíram um índice de transparência relacionado à publicação de previsões de inflação pelo banco central. Investigaram de que forma o grau de transparência na condução da política monetária afeta resultados 
macroeconômicos, em particular a inflação, o produto, e a variabilidade destes. Para tanto, foram usados dados cross-country para 87 países. Os resultados da análise mostram que um grau mais elevado de transparência está associado com inflação média mais baixa. Segundo os autores, a relação ocorre tanto em países com âncora nominal baseada em metas para inflação quanto em países que adotam âncora baseada em agregados monetários. Todavia, afirmam que em países onde a âncora é cambial o resultado não se aplica e que não há indício de que um aumento da transparência esteja ligado à maior volatilidade do produto.

Rafferty e Tomljanovich (2002) estudaram o efeito do aumento na transparência do Federal Reserve, resultante dos anúncios de política do Federal Open Market Committee (FOMC) a partir de 1994, sobre as expectativas do público. A principal variável no estudo é a taxa de juros dos títulos do Tesouro norte-americano e a sua previsibilidade pelo mercado financeiro. $\mathrm{O}$ resultado sugere que um maior grau de transparência da autoridade monetária está associado com maior eficiência e menor volatilidade nos mercados financeiros.

A relação entre a estrutura institucional da política monetária e o desempenho macroeconômico também é estudado por Cecchetti e Krause (2002). Levando em conta as informações presentes em Fry et al (2000), os autores sobreditos calcularam medidas de desempenho macroeconômico e eficiência política baseados na variabilidade da inflação e do produto, e medidas quantitativas para as características estruturais do banco central. Ademais, foram realizados testes para correlações simples e análise multivariada, com dados cross-country. Os resultados obtidos sugerem que a credibilidade, e em menor grau a transparência, são correlacionadas de maneira significativa com a variabilidade dos resultados macroeconômicos. Independência e accountability, por sua vez, não parecem explicar de forma significativa tais variáveis.

Demertzis e van der Cruijsen (2005) examinaram como a relação entre inflação e expectativas inflacionárias se altera com o incremento na transparência. Para mensurar a transparência utilizaram o índice elaborado por Eijffinger e Geraats (2004) construindo um subíndice para cada um dos tipos de transparência identificados por Geraats (2002). A soma destes subíndices é o índice total de transparência e permite avaliar a evolução da transparência ao longo do tempo. Com base nessa informação foi realizada uma análise por meio de dados em painel, assim como um exame específico para cada país estudado a fim de distinguir o impacto das diferentes mudanças institucionais na relação entre inflação e expectativas.

A amostra utilizada por Demertzis e van der Cruijsen (2005), além da Zona do Euro, contém dados de oito países industrializados. Segundo os autores, há evidência de que um aumento na transparência torna as expectativas de inflação do setor privado mais bem ancoradas. Os resultados ainda sugerem que alguns aspectos da transparência, tal como a transparência sobre as metas de inflação (transparência política), são mais importantes do que outros na construção das expectativas.

Bauer et al (2006) analisaram se a habilidade do público na previsão dos re- 
sultados macroeconômicos e das políticas futuras adotadas pelo Fed foi afetada pelas mudanças na comunicação com o setor privado a partir de 1994. Esse período foi selecionado porque foi quando o FOMC passou a fazer a divulgação de anúncios a respeito de mudanças na política monetária norte-americana. A análise concentra-se nas expectativas de curto e de longo prazo do setor privado sobre variáveis macroeconômicas como inflação, produto interno bruto, e desemprego. Foi verificada uma queda no erro idiossincrático apontando para expectativas mais sincronizadas a partir de 1994. Não obstante, há indicação de que o erro expectacional comum aumentou e tornou-se mais volátil depois do início da divulgação de anúncios das mudanças políticas feitas pelo FOMC.

Com o objetivo de avaliar o efeito da transparência econômica sobre a taxa de juros de curto prazo, de Mendonça e Simão Filho (2007) utilizaram dados sobre transparência e inflação (média 1997-1998), e expectativa dos agentes econômicos quanto ao sucesso na condução da política monetária (índice para 1998) presentes em Fry et alii (2000). Ademais, foi utilizada a taxa de juros interbancária (média 1997-1998) extraída do International Financial Statistics para a avaliação empírica. A metodologia aplicada baseou-se em correlações simples e análise multivariada, com dados cross-country para 45 países. Os resultados obtidos indicam que a transparência contribui para uma redução da inflação com uma taxa de juros mais baixa.

Em suma, os resultados empíricos presentes na literatura revelam que a transparência do banco central está relacionada à média da inflação, à variabilidade de variáveis macroeconômicas, e à volatilidade dos mercados financeiros. Ademais, observa-se que o efeito da transparência sobre as expectativas do setor privado não deve ser desprezado.

\section{UMA ANÁLISE PARA O CASO BRASILEIRO}

Em junho de 1999 foi instituído o regime de metas para inflação no Brasil. Com esse regime monetário o Banco Central do Brasil (BCB) passou a ter, na prática, independência operacional. Em outras palavras, a autoridade monetária tem liberdade para utilizar os instrumentos de política monetária de forma a alcançar a meta de inflação. Todavia, o BCB não possui independência total, pois cabe ao governo a definição das metas e das bandas de variação para a inflação.

No caso de a meta de inflação ser descumprida o presidente do BCB deve explicar-se publicamente, mediante carta aberta ao Ministro da Fazenda, informando os motivos para o descumprimento, as medidas que serão tomadas para que a inflação retorne ao intervalo de tolerância, e o tempo para que tais medidas tenham efeito. Além disso, o BCB fica incumbido da divulgação de um relatório de inflação trimestral, o qual deve avaliar o desempenho do regime de metas para inflação, os resultados das políticas implementadas, assim como apresentar previsões para a trajetória futura da inflação. Ademais, o BCB divulga expectativas de mercado 
sobre variáveis que interferem na condução da política monetária (inflação, produto, etc.).

De acordo com Mishkin (2004), a divulgação de informações mostra-se essencial para a construção da transparência e da credibilidade em economias emergentes. O compromisso com a transparência e a importância desta na condução da política monetária brasileira ficam claros no Relatório de Inflação de junho de 1999 (p. 5, grifo nosso):

"O regime, em processo de adoção, constitui estratégia mais adequada, no atual contexto, para a manutenção da estabilidade de preços, uma vez que torna a política monetária mais transparente, ao deixar claros os meios usados pelo Banco Central para atingir este objetivo. Ao mesmo tempo, evidencia as limitações da política monetária e a eventual inconsistência de políticas econômicas, aumentando o grau de comprometimento do governo com o processo de estabilização de preços.”

O interesse no incremento da transparência é evidente também no discurso de posse de Henrique Meirelles (atual presidente do BCB) de janeiro de 2003. Ou seja, apesar da mudança na presidência do país e na presidência do BCB durante a vigência do regime de metas para inflação, a prioridade da manutenção de preços estáveis e o interesse em aumentar o grau de transparência na condução da política monetária permaneceram.

"A elaboração de relatórios e a divulgação de estudos e pesquisas também são fundamentais, visando à transparência da atuação da instituição para a sociedade. (...) Assim, o Banco Central dará ênfase não apenas ao aperfeiçoamento das estatísticas e dos modelos, mas à transparência e à comunicação.” (p. 6, grifo nosso)

Portanto, não há dúvidas de que com a introdução do regime de metas para inflação no Brasil foram adotadas medidas para aumentar a transparência política, de procedimento, operacional, e de política, conforme o critério de classificação construído por Geraats (2002). Logo, torna-se relevante verificar se o esforço em desenvolver transparência no período alterou o comportamento de variáveis como: inflação, produto, emprego, e taxa de juros.

\section{Análise de estatísticas descritivas}

Esta seção tem como objetivo verificar se houve alteração no comportamento de variáveis macroeconômicas devido à adoção do regime de metas para inflação e ao conseqüente aumento da transparência na condução da política monetária. Para tanto, é realizada uma análise, por meio de estatísticas descritivas, das seguintes séries: taxa de juros mensal over-Selic (divulgada pelo $\mathrm{BCB}$ ); inflação mensal medida pelo Índice de Preços ao Consumidor Amplo (IPCA - divulgado pelo Instituto Brasileiro de Geografia e Estatística - IBGE); taxa de desemprego mensal da região 
metropolitana de São Paulo (divulgada pela Fundação SEADE/DIEESE - incluindo desemprego aberto e oculto); e o logaritmo natural do PIB real dessazonalizado (variável calculada a partir da série de PIB nominal disponibilizada pelo BCB).

Todas as séries têm periodicidade mensal com início em agosto de 1994 e término em outubro de 2006. A escolha para o ano e o mês de início da análise está relacionada à implementação do Plano Real (período marcado por relativa estabilidade de preços). Com o objetivo de verificar o comportamento das séries em consideração, antes e depois da adoção das metas para inflação, uma análise estatística levando em conta os períodos agosto de 1994-junho de 1999 e julho de 1999-outubro de 2006 foi realizada.

Os dados apresentados na tabela 1 revelam que depois da adoção das metas para inflação, as médias da inflação e taxa de juros caíram consideravelmente (queda de $29 \%$ e $44 \%$, respectivamente). Por outro lado, a taxa de desemprego sofreu um aumento de $15 \%$, enquanto que o crescimento econômico não apresentou mudança significativa (aumento de $1 \%$ ). Considerando-se a análise da volatilidade, observa-se que com a exceção do PIB (que permaneceu estável) todas as séries apresentaram queda. Uma possível justificativa para esse resultado é a diminuição da assimetria de informações entre o público e o BCB fazendo com que as decisões de política monetária causem menor impacto sobre as expectativas do setor privado.

Tabela 1: Média, Desvio padrão e Coeficiente de Variação

\begin{tabular}{l|ccc|ccc}
\hline \multirow{2}{*}{\multicolumn{1}{c|}{ Série }} & \multicolumn{3}{|c|}{ agosto de 1994 -junho de 1999 } & \multicolumn{3}{c}{ julho de 1999 -outubro de 2006 } \\
\cline { 2 - 7 } & Média & DP & CV & Média & DP & CV \\
\hline Inflação & 0,861 & 0,788 & 0,915 & 0,614 & 0,503 & 0,819 \\
Desemprego & 15,759 & 2,270 & 0,144 & 18,125 & 1,394 & 0,077 \\
Juros & 2,529 & 0,886 & 0,350 & 1,421 & 0,216 & 0,152 \\
PIB & 10,745 & 0,065 & 0,006 & 10,936 & 0,073 & 0,007 \\
\hline
\end{tabular}

Nota: DP - desvio padrão; e CV - coeficiente de variação.

A figura 1 mostra a evolução da volatilidade da taxa de inflação ao longo do período analisado. Para o cálculo da medida de volatilidade foi considerado o desvio da taxa de inflação para cada mês em relação às respectivas médias (dos dois períodos) elevado ao quadrado. Observa-se que há uma tendência para a inflação ser menos volátil depois da adoção do regime de metas para inflação e o conseqüente aumento na transparência da condução da política monetária. Deve-se ressaltar que o pico de variância entre o fim de 2002 e meados de 2003 (vide figura 1) está relacionado à crise de confiança do público em relação ao compromisso de o novo governo assegurar a política de estabilidade de preços e os contratos existentes. 


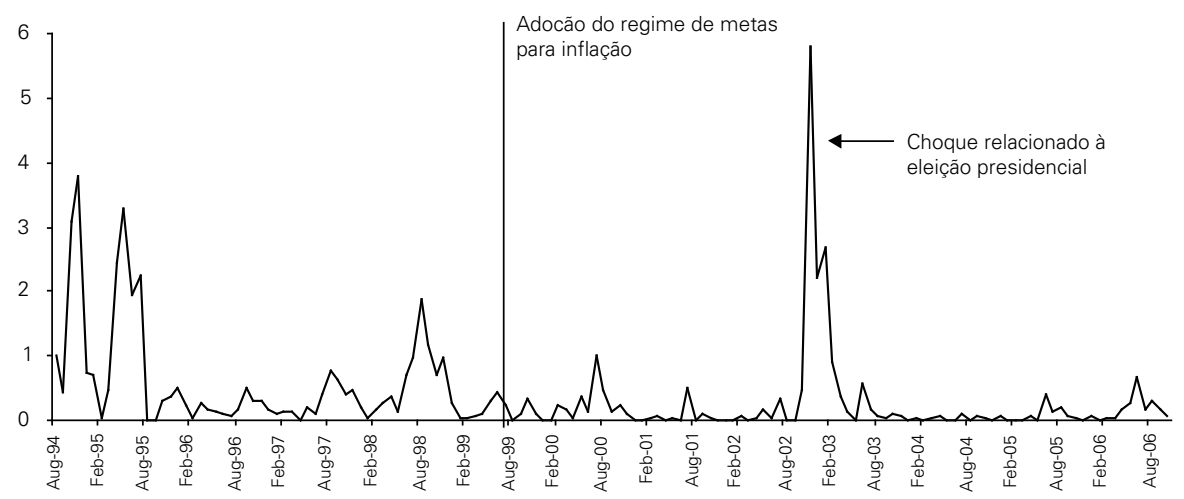

A figura 2 mostra a evolução da variabilidade da taxa de juros (over-Selic). O procedimento para a mensuração da volatilidade é análogo ao utilizado para o caso da inflação. Observa-se que a volatilidade torna-se menos acentuada depois da adoção do regime de metas para inflação. Este resultado é importante porque revela uma maior capacidade de planejamento do setor privado em relação à tomada de decisões. Uma possível conseqüência é que a combinação de uma menor taxa de juros e menor volatilidade possa estimular o setor privado a aumentar a taxa de investimento da economia. Deve-se notar que essa menor variabilidade pode estar relacionada ao fato de que a média da taxa de inflação e a sua variância também foram menores no período posterior à implantação do regime de metas. Além disso, tal como na análise para a inflação, o aumento da volatilidade observado no ano de 2003 é reflexo do processo eleitoral ocorrido no ano anterior.

Figura 2: Volatilidade da taxa de juros (over-Selic)

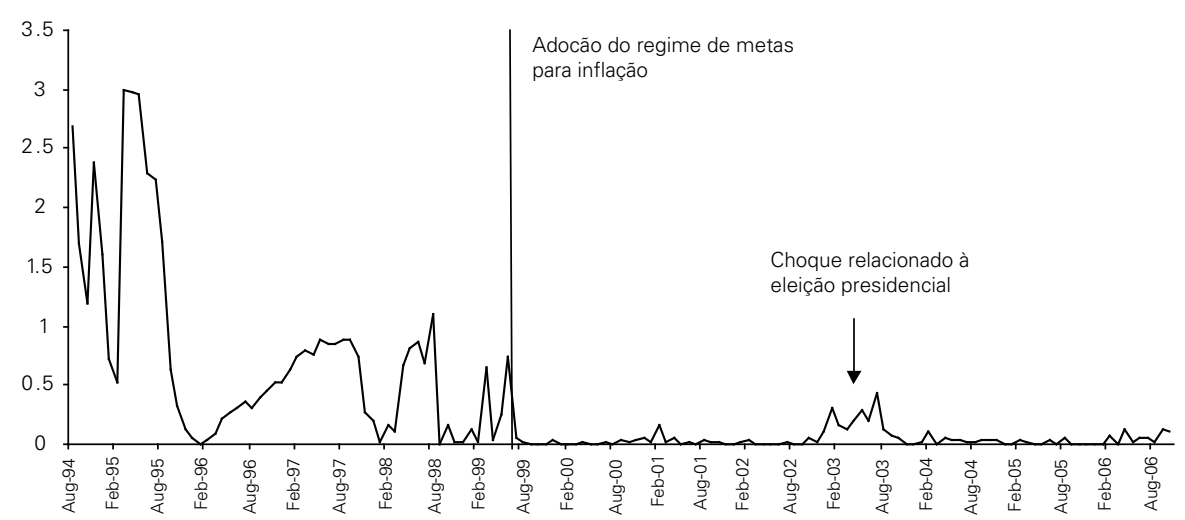

De forma semelhante ao procedimento adotado nos casos anteriores, a figura 3 mostra o comportamento da volatilidade da taxa de desemprego ao longo do 
período sob análise. Observa-se que, apesar de haver uma redução na volatilidade depois da adoção da estratégia de metas para inflação, não se pode dizer que a mesma tornou-se desprezível.

Figura 3: Volatilidade da taxa de desemprego

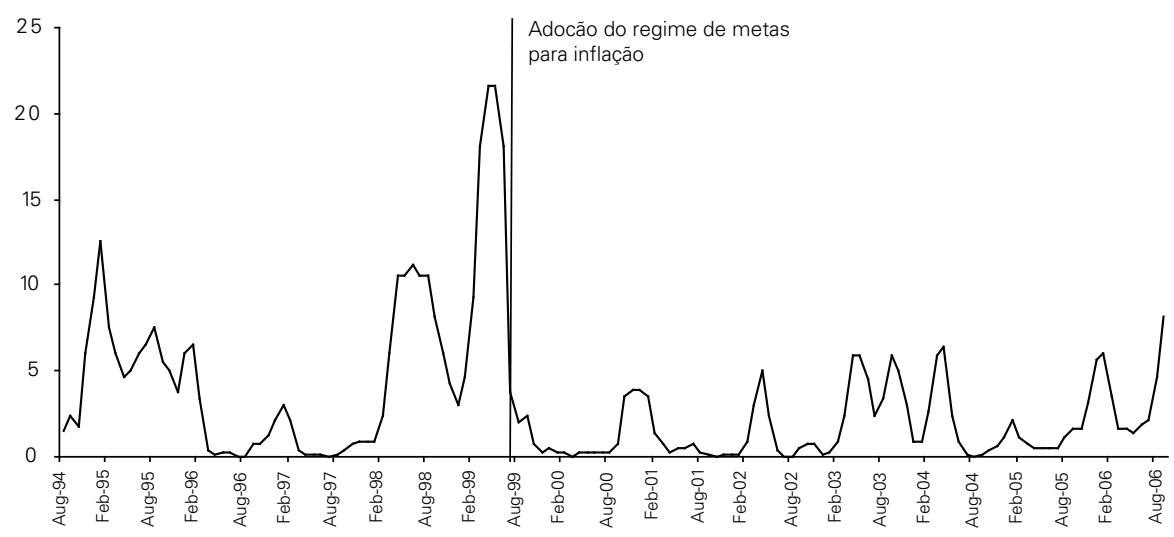

A evolução da volatilidade do PIB é mostrada na figura 4. Observa-se que não há uma mudança de comportamento significativa depois da adoção do regime de metas para inflação. Não obstante, verifica-se que a volatilidade reduziu-se até o ano de 2004 quando voltou a aumentar e apresentar uma trajetória semelhante à observada no início do segundo período (1999-2006). Tal como no caso da taxa de desemprego, a literatura sobre transparência não destaca efeitos sobre o produto. Uma justificativa é que a série do PIB apresenta características bastante particulares e está submetida a efeitos cíclicos diversos que explicam a trajetória dessa variável.

Com o objetivo de verificar o possível efeito do aumento da transparência do $\mathrm{BCB}$ sobre as quatro variáveis macroeconômicas em consideração, uma análise da distribuição das séries foi feita. Esta análise é relevante porque, no caso de as séries estarem próximas de uma distribuição normal isso significa um comportamento mais prognosticado das mesmas. Logo, há uma melhora na capacidade de previsão dos agentes econômicos sobre o comportamento das variáveis macroeconômicas, o que, por conseguinte, contribui para o processo de tomada de decisões. Para tanto, da mesma forma que a análise presente na tabela 1 , as séries foram divididas em dois períodos e submetidas a testes de assimetria e curtose da distribuição, além do teste de Jarque Bera (vide tabela 2). A questão a ser colocada é se, com a introdução do regime de metas para inflação e o conseqüente aumento da transparência da autoridade monetária, as séries analisadas apresentam um comportamento que permita aos agentes econômicos prever de forma mais eficiente o comportamento destas variáveis. 


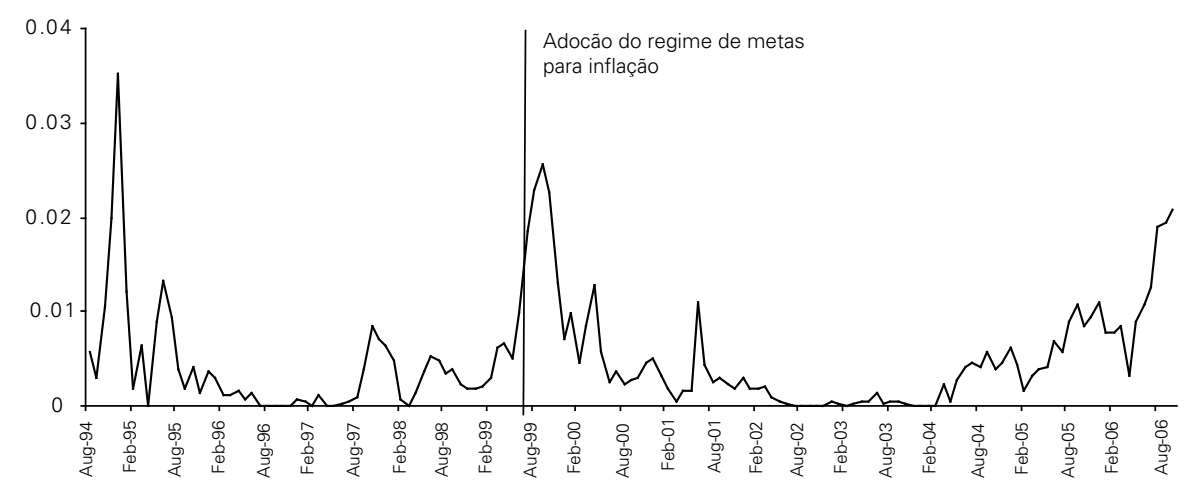

A tabela 2 revela que a distribuição da inflação está mais próxima de uma distribuição normal antes do incremento da comunicação do banco central com o público do que depois. Esse resultado pode estar relacionado às características particulares deste regime monetário no Brasil. Os vários descumprimentos da meta de inflação e os ajustes nas bandas de flutuação, além dos diversos choques sobre a economia desde meados de 1999, quando as metas para inflação foram introduzidas, podem ter contribuído para que os agentes econômicos não tivessem a percepção do comportamento esperado em um cenário de maior transparência. Ademais, este resultado pode estar relacionado ao próprio processo de desinflação ocorrido na economia brasileira desde a adoção das metas para inflação.

Tabela 2: Medidas de normalidade

\begin{tabular}{l|cc|cc|cc|cc}
\hline & \multicolumn{2}{|c|}{ Inflação } & \multicolumn{2}{c|}{ Juros } & \multicolumn{2}{c|}{ Desemprego } & \multicolumn{2}{c}{ PIB } \\
\cline { 2 - 9 } & A & D & A & D & A & D & A & D \\
\hline Assimetria & 0,758 & 2,031 & 0,665 & 0,774 & 0,379 & 0,025 & $-0,604$ & $-0,011$ \\
Curtose & 2,888 & 9,126 & 2,094 & 3,558 & 2,021 & 2,375 & 2,817 & 2,277 \\
\hline Jarque Bera & 5,676 & 198,118 & 6,371 & 9,917 & 3,768 & 1,442 & 3,671 & 1,919 \\
Probabilidade & $(0,059)$ & $(0,000)$ & $(0,041)$ & $(0,007)$ & $(0,152)$ & $(0,486)$ & $(0,160)$ & $(0,383)$ \\
\hline
\end{tabular}

Nota: $A$ = antes das metas para inflação, $D=$ depois das metas para inflação.

Os testes de assimetria e curtose para a taxa de juros não mostram uma tendência bem definida. As estatísticas Jarque Bera não apontam para distribuição normal a $5 \%$ de significância. Para a taxa de desemprego, os testes sugerem uma distribuição mais simétrica e a curtose mais próxima de três depois da introdução do regime de metas para inflação. Este resultado indica que a taxa de desemprego está mais próxima de uma tendência e pode ser prevista pelos agentes de forma mais eficiente em um cenário de maior transparência. 
Os testes para PIB e desemprego indicam uma distribuição mais simétrica depois da adoção do novo regime monetário. Os resultados das variáveis relacionadas à atividade real da economia podem estar associados ao incentivo dado aos formuladores de política monetária em um cenário de maior transparência. Quando a diferença entre a inflação observada e a inflação esperada é menor, há menos oscilação nas variáveis reais da economia. Assim, considerando esta perspectiva válida, a conseqüência é um menor incentivo para promover políticas de estabilização por meio da manifestação do viés inflacionário devido ao aumento na $a c$ countability. Como conseqüência, o PIB e a taxa de desemprego tornam-se mais estáveis.

É importante notar que os choques externos concernentes às economias emergentes na década de 1990, o processo de desinflação da economia depois do Plano Real, os choques relacionados às crises energética em 2001 e eleitoral em 2002 e 2003, o ambiente internacional de negócios mais favorável a partir de 2003, dentre outros fatores que atuaram sobre a economia brasileira no período analisado, possivelmente influenciaram as variáveis macroeconômicas utilizadas na análise anterior. Logo, com o objetivo de verificar a consistência dos resultados obtidos, a análise estatística das séries foi refeita levando em conta as séries obtidas por meio da aplicação de médias móveis (três períodos à frente e três períodos atrás) e do filtro Hodrick-Prescott (HP) (vide tabela 3). A idéia é que dessa forma são eliminadas as volatilidades em torno da tendência das séries e, portanto, o efeito causado pelos choques é eliminado. Destarte, é possível avaliar com mais clareza se houve mudança (de fato) no comportamento de cada variável antes e depois da introdução das metas para inflação.

Os resultados contidos na tabela 3 sugerem poucas alterações em relação àqueles presentes nas tabelas 1 e 2 . Observa-se que as médias do IPCA e da taxa de juros diminuíram, e as volatilidades de todas as séries, com exceção do PIB, decresceram depois do aumento da transparência. A análise de normalidade também não mostra mudanças relevantes. Isto é, as variáveis suavizadas apresentam tendência semelhante às variáveis originais. Ver Tabela 3 na página seguinte

Tabela 3: Estatística descritiva - Médias Móveis e Filtro HP

\begin{tabular}{|c|c|c|c|c|c|c|c|c|}
\hline \multirow{2}{*}{$\begin{array}{l}\text { Médias } \\
\text { móveis }\end{array}$} & \multicolumn{2}{|c|}{ Inflação } & \multicolumn{2}{|c|}{ Juros } & \multicolumn{2}{|c|}{ Desemprego } & \multicolumn{2}{|r|}{ PIB } \\
\hline & A & $\mathrm{D}$ & A & $D$ & A & $\mathrm{D}$ & A & $\mathrm{D}$ \\
\hline Média & 0,805 & 0,625 & 2,451 & 1,433 & 15,858 & 18,214 & 10,750 & 10,931 \\
\hline Desvio-padrão & 0,630 & 0,329 & 0,754 & 0,178 & 2,170 & 1,181 & 0,051 & 0,067 \\
\hline Assimetria & 0,687 & 2,090 & 0,974 & 0,965 & 0,288 & 0,066 & $-0,311$ & 0,036 \\
\hline Curtose & 2,226 & 7,574 & 2,569 & 3,401 & 1,789 & 1,770 & 1,684 & 1,986 \\
\hline \multirow[t]{2}{*}{ Jarque-Bera } & 5,797 & 135,965 & 9,292 & 13,753 & 4,197 & 5,424 & 4,941 & 3,661 \\
\hline & $(0,055)$ & $(0,000)$ & $(0,010)$ & $(0,001)$ & $(0,123)$ & $(0,066)$ & $(0,085)$ & $(0,160)$ \\
\hline
\end{tabular}




\begin{tabular}{|c|c|c|c|c|c|c|c|c|}
\hline \multirow[t]{2}{*}{ Filtro HP } & \multicolumn{2}{|c|}{ IPCA } & \multicolumn{2}{|c|}{ SELIC } & \multicolumn{2}{|c|}{ Desemprego } & \multicolumn{2}{|c|}{ PIB } \\
\hline & $A$ & D & A & D & $A$ & $\mathrm{D}$ & $A$ & $\mathrm{D}$ \\
\hline Média & 0,861 & 0,614 & 2,529 & 1,421 & 15,759 & 18,125 & 10,745 & 10,936 \\
\hline Desvio-padrão & 0,623 & 0,178 & 0,640 & 0,080 & 2,092 & 0,882 & 0,054 & 0,070 \\
\hline Assimetria & 0,632 & $-0,806$ & 1,045 & $-0,559$ & 0,327 & $-1,078$ & $-0,198$ & 0,085 \\
\hline Curtose & 2,030 & 2,896 & 2,683 & 2,951 & 1,848 & 3,527 & 1,778 & 1,901 \\
\hline \multirow[t]{2}{*}{ Jarque-Bera } & 6,243 & 9,559 & 10,990 & 4,587 & 4,309 & 18,070 & 4,057 & 4,534 \\
\hline & $(0,044)$ & $(0,008)$ & $(0,004)$ & $(0,101)$ & $(0,116)$ & $(0,000)$ & $(0,132)$ & $(0,104)$ \\
\hline
\end{tabular}

Como proxy para as expectativas das variáveis que estão sendo analisadas foram utilizadas as respectivas médias móveis. O motivo para a aplicação deste procedimento se deve ao fato de que o $\mathrm{BCB}$ começou a disponibilizar as séries para expectativas de variáveis macroeconômicas apenas a partir de 1999 e que os agentes econômicos possuem um horizonte limitado de informações quando formam suas expectativas. Os resultados indicam que há menor incerteza na formação de expectativas pelo público depois do aumento da transparência na condução da política monetária.

Dado que o filtro HP aponta uma tendência de longo prazo, a redução na volatilidade das séries filtradas pode ser interpretada como uma menor volatilidade no comportamento de longo prazo das séries estudadas. Logo, há indícios de um cenário econômico menos incerto depois da introdução do regime de metas para inflação. Esta mudança pode estar relacionada à maior transparência das ações da autoridade monetária. A justificativa é que em um ambiente de menor assimetria informacional as expectativas são mais acuradas e mais confiáveis, assim como as trajetórias de longo prazo das variáveis macroeconômicas mais previsíveis.

\section{O impacto da transparência sobre variáveis macroeconômicas}

Com o objetivo de dar maior robustez à análise realizada, esta seção avalia o impacto da opacidade (ausência de transparência) sobre as variáveis macroeconômicas e a significância deste impacto. Além disso, é feito um teste de quebra estrutural, com uso de variável dummy, para verificar se houve alterações estruturais nas séries quando da implantação do regime de metas para inflação em junho de 1999.

Para efetuar a análise do impacto da transparência sobre as variáveis macroeconômicas foram construídos indicadores de opacidade e realizadas estimações por meio do método dos Mínimos Quadrados Ordinários para as quatro variáveis sob consideração. O indicador de opacidade é obtido pela diferença entre a variável esperada (obtido por meio de médias móveis) e a variável observada. Supõe-se que, quanto maior o volume de informações do público a respeito dos dados econômicos, mais próximas da realidade serão suas expectativas e, portanto, menor será o indicador de opacidade. 
Os indicadores de opacidade aparecem nas regressões com um período (um mês) de defasagem. Espera-se que os efeitos da opacidade em relação às variáveis em um dado mês só sejam percebidos no mês posterior. Para evitar espuriedade nos resultados das estimações foi verificada a presença de raiz unitária em todas as séries por meio do teste ampliado de Dickey-Fuller (ADF). Os resultados indicam que, com exceção da taxa de desemprego (I(1)), as demais séries são estacionárias em nível (vide apêndice A.1). Foram incluídas defasagens de um período em todos os modelos. Tal procedimento tem como base a hipótese de exogeneidade estrita das variáveis independentes. Esta hipótese seria claramente violada sem as defasagens devido à influência contemporânea que as séries defasadas têm em cada uma das variáveis em consideração. Ademais, foram introduzidas dummies pontuais nas regressões segundo os choques existentes e com base na análise dos resíduos dos modelos originais.

Os testes de quebra estrutural foram realizados com a utilização de uma variável dummy, cujo valor é 1 no período anterior a junho de 1999 (incluindo este mês) e 0 no período posterior a este mês. Esta data foi definida como ponto de mudança estrutural porque é o mês da implementação do regime de metas para inflação no país. Os resultados obtidos indicam que a hipótese de mudança estrutural é confirmada para as variáveis taxa de juros e PIB, e não confirmada para a taxa de desemprego e a taxa de inflação (vide tabela 4).

Apesar da maior transparência é provável que a quebra estrutural para taxa de inflação tenha ocorrido mais tarde devido à crise eleitoral em 2002 e início de 2003. Ademais, os vários descumprimentos das metas estabelecidas, ocorridos até 2003, podem ter colaborado para a desconfiança do público em relação ao compromisso com o objetivo de política monetária. Em relação à taxa de desemprego, uma possível explicação para não detectar a quebra estrutural se deve à abertura comercial do país que culminou com uma elevação nessa taxa ao longo dos anos 1990.

Tabela 4: Modelos de Quebra Estrutural

\begin{tabular}{|c|c|c|c|c|}
\hline Variável Dependente & INF & coeficiente & estatística t & p-valor \\
\hline \multirow[t]{8}{*}{ Regressores } & $\mathrm{C}$ & 0,019 & 0,405 & 0,686 \\
\hline & INF(-1) & 0,939 & 13,431 & 0,000 \\
\hline & OP_INF(-1) & 0,731 & 6,419 & 0,000 \\
\hline & D1 & $-0,016$ & $-0,314$ & 0,754 \\
\hline & OP_INF(-1)*D1 & $-0,121$ & $-0,966$ & 0,336 \\
\hline & D2 & 1,555 & 17,699 & 0,000 \\
\hline & $\mathrm{R}^{2}$ ajustado & 0,699 & Estatística F & 65,595 \\
\hline & N. OBS. & 140 & Prob(Estat F) & $(0,000)$ \\
\hline Variável Dependente & JUROS & coeficiente & estatística t & p-valor \\
\hline \multirow[t]{3}{*}{ Regressores } & $\mathrm{C}$ & 0,034 & 0,624 & 0,534 \\
\hline & JUROS(-1) & 0,968 & 25,704 & 0,000 \\
\hline & OP_JUROS(-1) & 1,080 & 5,189 & 0,000 \\
\hline
\end{tabular}




\begin{tabular}{|c|c|c|c|c|}
\hline & D1 & $-0,081$ & $-1,905$ & 0,059 \\
\hline & OP_JUROS(-1)*D1 & $-0,689$ & $-2,710$ & 0,008 \\
\hline & D3 & 0,828 & 14,122 & 0,000 \\
\hline & D4 & 1,162 & 11,026 & 0,000 \\
\hline & $R^{2}$ ajustado & 0,966 & Estatística F & 662,807 \\
\hline & N. OBS. & 140 & Prob(Estat F) & $(0,000)$ \\
\hline Variável Dependente & $\triangle \mathrm{DES}$ & coeficiente & estatística t & p-valor \\
\hline \multirow[t]{7}{*}{ Regressores } & $\mathrm{C}$ & $-0,002$ & $-0,103$ & 0,918 \\
\hline & $\Delta \mathrm{DES}(-1)$ & 0,789 & 19,321 & 0,000 \\
\hline & OP_DES(-1) & 0,848 & 21,409 & 0,000 \\
\hline & D1 & 0,025 & 0,748 & 0,456 \\
\hline & OP_DES(-1)*D1 & $-0,093$ & $-1,543$ & 0,125 \\
\hline & $\mathrm{R}^{2}$ ajustado & 0,701 & Estatística F & 82,552 \\
\hline & N. OBS. & 140 & Prob(Estat F) & $(0,000)$ \\
\hline Variável Dependente & PIB & coeficiente & estatística t & $\mathrm{p}$-valor \\
\hline \multirow[t]{8}{*}{ Regressores } & $C$ & $-0,274$ & $-0,873$ & 0,384 \\
\hline & $\mathrm{PIB}(-1)$ & 1,025 & 35,703 & $0,000 * * *$ \\
\hline & OP_PIB(-1) & 0,885 & 10,575 & $0,000 * * *$ \\
\hline & D1 & 0,004 & 0,568 & 0,571 \\
\hline & OP_PIB(-1)*D1 & $-0,268$ & $-2,719$ & $0,007^{* *}$ \\
\hline & D5 & 0,140 & 35,842 & $0,000 * * *$ \\
\hline & $\mathrm{R}^{2}$ ajustado & 0,973 & Estatística F & 1009,958 \\
\hline & N. OBS. & 140 & Prob(Estat F) & $(0,000)$ \\
\hline
\end{tabular}

Nota: INF: taxa de inflação mensal; OP_INF: indicador de opacidade sobre a inflação; JUROS: taxa de juros mensal (over-Selic); OP_JUROS: indicador de opacidade sobre a taxa de juros; $\triangle \mathrm{DES}$ : primeira diferença da taxa de desemprego mensal; OP_DES: indicador de opacidade sobre a taxa de desemprego; PIB: logaritmo natural do PIB real mensal; OP_PIB: indicador de opacidade sobre o PIB. D1: dummy de quebra estrutural (1, para valores entre agosto de 1994 a junho de 1999; 0 caso contrário); D2: dummy para choque sobre a inflação relacionado à crise eleitoral (1 em novembro de 2002; 0 caso contrário); D3: dummy para choques sobre a taxa de juros relacionados às crises nos países emergentes (1 em março de 1995, setembro e outubro de 1998; 0 caso contrário); D4: dummy para choques sobre a taxa de juros relacionados às crises nos países emergentes (1 em novembro de 1997 e março de 1999; 0 caso contrário); D5: dummy para choque sobre o produto (1 em março de 1995; 0 caso contrário).

A tabela 4 revela que os indicadores de opacidade são significativos nos modelos para todo o período. Em todas as regressões os sinais dos coeficientes dos indicadores sugerem que quanto maior a opacidade (menor transparência) maiores serão as variáveis analisadas. Ou seja, conforme indicado na análise da seção anterior, menor transparência aumenta a taxa de inflação e de juros. Por outro lado, quanto menor a transparência maior a variação da taxa de desemprego e maior o PIB. Estes diagnósticos também são similares aos do exame das médias.

Devido à possibilidade de alteração estrutural, o exame para dois momentos distintos foi realizado. Os dados foram divididos em dois períodos tal como na análise das estatísticas descritivas (agosto/1994 - junho/1999; e julho/1999 outubro/2006) e novas regressões foram realizadas (vide tabela 5). Procurou-se 
verificar se houve mudança significativa no coeficiente do indicador de opacidade entre os períodos.

É importante salientar que foram realizados testes de raiz unitária (ADF) para todas as séries em ambos os períodos. No período anterior à introdução do regime de metas para inflação o resultado obtido indica que, com exceção da taxa de juros (que é I(1)), todas as séries são estacionárias em nível. Para o período posterior à adoção das metas foi observado que as séries referentes à taxa de juros e ao desemprego são I(1), enquanto que as demais são estacionárias em nível (vide apêndice A.1).

Os resultados referentes aos coeficientes dos indicadores de opacidade das variáveis nos dois períodos ratificam aqueles encontrados para o período total. Com a exceção do coeficiente do indicador de opacidade para a taxa de juros, verifica-se que não há variação expressiva dos coeficientes entre os dois períodos e que os mesmos apresentam significância estatística em todos os modelos. Por conseguinte, há indicação de que o nível de opacidade, ou grau de transparência sobre os dados econômicos, afete as variáveis macroeconômicas em ambos os períodos. As pequenas alterações observadas (aumento nos coeficientes com a maior transparência do $\mathrm{BCB}$ ) sugerem que a opacidade é mais relevante para explicar o nível das variáveis macroeconômicas no período em que ela é menor. Deve-se notar que a variação da taxa de juros mostrou-se positivamente relacionada ao indicador de opacidade. Em outras palavras, quanto maior a opacidade (menor transparência) maior a variabilidade da taxa de juros.

Tabela 5: Modelos com Indicadores de Opacidade

\begin{tabular}{|c|c|c|c|c|c|c|c|c|}
\hline \multicolumn{5}{|c|}{ Período novembro/1994 a junho/1999 } & \multicolumn{4}{|c|}{ Período julho/1999 a outubro/2006 } \\
\hline $\begin{array}{c}\text { Variável } \\
\text { Dependente }\end{array}$ & INF & Coeficiente & estatística t & $\mathrm{p}$-valor & INF & coeficiente & estatística t & p-valor \\
\hline \multirow[t]{7}{*}{ Regressores } & C & 0,000 & $-0,005$ & 0,996 & $\mathrm{C}$ & 0,000 & $-0,002$ & 0,999 \\
\hline & INF(-1) & 0,913 & 12,484 & 0,000 & INF(-1) & 0,935 & 9,403 & 0,000 \\
\hline & OP_INF(-1) & 0,566 & 3,815 & 0,000 & OP_INF(-1) & 0,657 & 4,469 & 0,000 \\
\hline & D6 & 0,664 & 2,702 & 0,009 & D7 & 1,168 & 4,216 & 0,000 \\
\hline & & & & & D8 & 1,601 & 5,424 & 0,000 \\
\hline & $\mathrm{R}^{2}$ ajustado & 0,773 & Estatística F & 62,261 & $\mathrm{R}^{2}$ ajustado & 0,708 & Estatística F & 51,208 \\
\hline & N. OBS. & 55 & Prob(Estat F) & 0,000 & N. OBS. & 84 & Prob(Estat F) & 0,000 \\
\hline $\begin{array}{c}\text { Variável } \\
\text { Dependente }\end{array}$ & $\triangle \mathrm{JUROS}$ & Coeficiente & estatística t & $\mathrm{p}$-valor & $\triangle J U R O S$ & Coeficiente & estatística t & $\mathrm{p}$-valor \\
\hline \multirow[t]{7}{*}{ Regressores } & C & $-0,084$ & $-2,762$ & 0,008 & C & $-0,013$ & $-1,208$ & 0,231 \\
\hline & $\triangle J U R O S(-1)$ & 0,271 & 3,734 & 0,001 & $\triangle J U R O S(-1)$ & 0,247 & 3,534 & 0,001 \\
\hline & OP_JUROS(-1) & 0,731 & 4,759 & 0,000 & OP_JUROS(-1) & 1,669 & 12,581 & 0,000 \\
\hline & D9 & 0,657 & 6,218 & 0,000 & D13 & 0,265 & 23,069 & 0,000 \\
\hline & D10 & 1,075 & 12,235 & 0,000 & & & & \\
\hline & D11 & 0,489 & 5,159 & 0,000 & & & & \\
\hline & D12 & 0,918 & 20,627 & 0,000 & & & & \\
\hline
\end{tabular}




\begin{tabular}{|c|c|c|c|c|c|c|c|c|}
\hline & $\mathrm{R}^{2}$ ajustado & 0,752 & Estatística F & 28,257 & $\mathrm{R}^{2}$ ajustado & 0,668 & Estatística F & 56,031 \\
\hline & N. OBS. & 55 & Prob(Estat F) & 0,000 & N. OBS. & 83 & Prob(Estat F) & 0,000 \\
\hline $\begin{array}{c}\text { Variável } \\
\text { Dependente }\end{array}$ & DES & Coeficiente & estatística $\mathrm{t}$ & $\mathrm{p}$-valor & $\triangle \mathrm{DES}$ & Coeficiente & estatística t & $\mathrm{p}$-valor \\
\hline \multirow[t]{5}{*}{ Regressores } & C & $-0,393$ & $-0,682$ & 0,498 & C & $-0,003$ & $-0,147$ & 0,884 \\
\hline & DES(-1) & 1,032 & 27,768 & 0,000 & $\triangle \mathrm{DES}(-1)$ & 0,769 & 14,205 & 0,000 \\
\hline & OP_DES(-1) & 0,451 & 4,372 & 0,000 & OP_DES(-1) & 0,830 & 20,499 & 0,000 \\
\hline & $\mathrm{R}^{2}$ ajustado & 0,963 & Estatística F & 701,316 & $\mathrm{R}^{2}$ ajustado & 0,671 & Estatística F & 84,599 \\
\hline & N. OBS. & 55 & Prob(Estat F) & 0,000 & N. OBS. & 83 & Prob(Estat F) & 0,000 \\
\hline $\begin{array}{c}\text { Variável } \\
\text { Dependente }\end{array}$ & PIB & Coeficiente & estatística t & $\mathrm{p}$-valor & PIB & Coeficiente & estatística t & $\mathrm{p}$-valor \\
\hline \multirow[t]{6}{*}{ Regressores } & C & $-0,747$ & $-0,951$ & 0,346 & C & 0,056 & 0,231 & 0,818 \\
\hline & $\mathrm{PIB}(-1)$ & 1,070 & 14,647 & 0,000 & $\mathrm{PIB}(-1)$ & 0,995 & 44,811 & 0,000 \\
\hline & OP_PIB(-1) & 0,670 & 7,314 & 0,000 & OP_PIB(-1) & 0,765 & 6,932 & 0,000 \\
\hline & D14 & 0,143 & 20,288 & 0,000 & D15 & $-0,059$ & $-4,368$ & 0,000 \\
\hline & $\mathrm{R}^{2}$ ajustado & 0,870 & Estatística F & 121,196 & $\mathrm{R}^{2}$ ajustado & 0,962 & Estatística F & 699,404 \\
\hline & N. OBS. & 55 & Prob(Estat F) & 0,000 & N. OBS. & 84 & Prob(Estat F) & 0,000 \\
\hline
\end{tabular}

Nota: DES: taxa de desemprego mensal (SEADE/DIEESE); $\triangle J U R O S$ : primeira diferença da taxa de juros mensal (over-Selic); D6: dummy para choques sobre a inflação relacionados às crises nos países emergentes (1 em outubro de 1994, abril de 1995 e fevereiro de 1999; 0 caso contrário); D7: dummy para choque sobre a inflação relacionado à crise energética (1 em julho de 2000; 0 caso contrário); D8: dummy para choque sobre a inflação relacionado à crise eleitoral (1 em novembro de 2002; 0 caso contrário); D9: dummy para choque sobre a taxa de juros relacionado à crise nos países emergentes (1 em março de 1995; 0 caso contrário); D10: dummy para choque sobre a taxa de juros relacionado à crise nos países emergentes (1 em novembro de 1997; 0 caso contrário); D11: dummy para choque sobre a taxa de juros relacionado à crise nos países emergentes (1 em outubro de 1998; 0 caso contrário); D12: dummy para choque sobre a taxa de juros relacionado à crise nos países emergentes (1 em março de 1999; 0 caso contrário); D13: dummy para choque sobre a taxa de juros relacionado à crise eleitoral (1 em janeiro e julho de 2003; 0 caso contrário); D14: dummy para choque sobre o PIB (1 em março de 1995; 0 caso contrário); D15: dummy para choque sobre o PIB (1 em junho de 2001; 0 caso contrário).

\section{CONSIDERAÇÕES FINAIS}

As mudanças na comunicação do $\mathrm{BCB}$ com o público depois da adoção do regime de metas para inflação apontam para um aumento no grau de transparência. Os resultados encontrados sugerem que houve uma redução na média e na volatilidade da inflação depois do aumento da transparência na condução da política monetária. Além disso, houve diminuição na volatilidade da taxa de juros, o que por sua vez indica uma maior eficiência na previsão do mercado em relação às ações do banco central.

Outro resultado positivo da análise realizada para a economia brasileira refere-se à diminuição da variabilidade da taxa de desemprego depois do incremento na comunicação da autoridade monetária com o público. Soma-se a esta observação o fato de a volatilidade do PIB não ter aumentado de forma significativa depois de 1999. Portanto, há indicações de que a estabilidade econômica é maior quando a incerteza acerca do comportamento do banco central e de suas intenções é menor. Um ambiente econômico mais estável é benéfico, pois torna o agente econômico 
mais confiante para tomar decisões de investimento e consumo. Logo, há uma melhora no comportamento das variáveis macroeconômicas que pode contribuir para um aumento no crescimento econômico.

A análise da distribuição das variáveis nos dois períodos sugere que o aumento da transparência não aproximou as taxas de inflação e de juros de uma distribuição normal. O próprio processo de desinflação, os choques sobre a economia, e as peculiaridades na condução do regime de metas para inflação no Brasil podem representar empecilhos para uma melhor previsão do comportamento dessas variáveis. Entretanto, a diminuição da assimetria nas distribuições das variáveis reais analisadas (PIB e taxa de desemprego) e a aproximação de uma distribuição normal depois do aumento da transparência (adoção das metas para inflação), podem indicar que tais variáveis estão mais próximas de suas tendências permitindo assim uma melhor previsão pelo público. As estimações realizadas com indicadores de opacidade confirmaram grande parte dos resultados obtidos no exame por meio de estatísticas descritivas. Em suma, maior transparência contribui para reduzir a inflação e a taxa de juros (inclusive sua volatilidade), além de reduzir a variação da taxa de desemprego.

\section{REFERÊNCIAS BIBLIOGRÁFICAS}

BANCO CENTRAL DO BRASIL (1999) Relatório de Inflação, v. 1, n. 1, Junho. Disponível em http:// www.bcb.gov.br/?RELINF.

BAUER, A.; EISENBEIS, R. A.; WAGGONER, D. F.; ZHA, T. (2006) "Transparency, expectations, and forecasts." Economic Review, Federal Reserve Bank of Atlanta, 1. ${ }^{\circ}$ trimestre, 1-25.

CARNEIRO, F. G.; DIVINO, J. A.; ROCHA, C. H. (2003) Reconsiderando o efeito Fisher: uma análise de cointegração entre taxas de juros e inflação. Revista Nova Economia, v. 13, n. 1, 81-100.

CECHETTI, S. G.; KRAUSE, S. (2002) “Central Bank structure, policy efficiency, and macroeconomic performance: exploring empirical relationships.” Review, Federal Reserve Bank of St. Louis, 47-59.

CHORTAREAS, G.; STASAVAGE, D.; STERNE, G. (2002) "Does it pay to be transparent? International evidence from central bank forecasts.” Review, Federal Reserve Bank of St. Louis, July-Aug., v. 84, n. 4, 99-118.

de MENDONÇA, H.F. e SIMÃO FILHO, J. (2007) "Economic Transparency and Effectiveness of Monetary Policy" Journal of Economic Studies, v. 34, n. 6, 497-514.

DEMERTZIS, M.; van der CRUIJSEN, C. (2005) "The impact of Central Bank transparency on inflation expectations." De Nederlandsche Bank Working Paper $n .^{\circ}$ 031/2005, Março.

EIJFFINGER, S. C. W.; GERAATS, P. (2004) "How transparent are Central Banks?” Cambridge Working Papers in Economics, n. ${ }^{\circ}$ 0411, January.

FAUST, J.; SVENSSON, L. E. O. (2001) "Transparency and credibility: monetary policy with unobservable goals." International Economic Review, v. 42, n. 2, 369-397.

FRY, M.; JULIUS, D.; MAHADEVA, L.; ROGER, S.; STERNE, G. (2000) "Key issues in the choice of monetary policy framework.” In: MAHADEVA, Lavan; STERNE, Gabriel. (Eds.). Monetary policy frameworks in a global context. London: Routledge, 1-216.

GERAATS, P. M. (2002) “Central Bank transparency.” Economic Journal, v. 112, 532-565.

JENSEN, H. (2002) "Optimal degrees of transparency in monetary policymaking." Scandinavian Journal of Economics, v. 104, n. 3, 399-422.

MEIRELLES, H. (2003) Discurso de posse na presidência do Banco Central do Brasil, Jan. 2003. Disponível em http://www.bcb.gov.br/?PRONUNC.

MISHKIN, F.S. (1992) Is the Fisher effect for real? A reexamination of the relationship between inflation and interest rates. Journal of Monetary Economics, v. 30, 195-215. 
MISHKIN, F. S. (2004) “Can Central Bank transparency go too far?” NBER Working Paper 10829, October.

RAFFERTY, M.; TOMLJANOVICH, M. (2002) “Central Bank transparency and market efficiency: an econometric analysis." Journal of Economics and Finance, v. 26, n. 2, 150-161.

WALSH, C. (2003) “Accountability, transparency, and inflation targeting.” Journal of money, Credit and Banking, v. 35, n. 5, 829-849.

\section{APÊNDICE}

A.1. Testes de Raiz Unitária (ADF)

\begin{tabular}{|c|c|c|c|c|c|c|}
\hline & Séries & Def. & Teste & $\begin{array}{c}\text { Valor crítico } \\
1 \% \\
\end{array}$ & $\begin{array}{c}\text { Valor crítico } \\
5 \% \\
\end{array}$ & $\begin{array}{c}\text { Valor críticc } \\
10 \% \\
\end{array}$ \\
\hline \multirow{9}{*}{$\begin{array}{l}\text { ago/1994 a } \\
\text { out/2006 }\end{array}$} & INF & 0 & -4.588 & -3.476 & -2.881 & -2.577 \\
\hline & JUROS & 0 & -3.027 & -3.476 & -2.881 & -2.577 \\
\hline & DES & 11 & -2.474 & -3.479 & -2.883 & -2.578 \\
\hline & $\triangle \mathrm{DES}$ & 10 & -2.392 & -2.582 & -1.943 & -1.615 \\
\hline & PIB & 0 & -5.959 & -4.022 & -3.441 & -3.145 \\
\hline & OP_INF & 1 & -11.259 & -2.582 & -1.943 & -1.615 \\
\hline & OP_JUROS & 2 & -10.464 & -2.582 & -1.943 & -1.615 \\
\hline & OP_DES & 8 & -9.394 & -2.583 & -1.943 & -1.615 \\
\hline & OP_PIB & 6 & -8.951 & -2.582 & -1.943 & -1.615 \\
\hline \multirow{9}{*}{$\begin{array}{l}\text { ago/1994 a } \\
\text { jun/1999(2) }\end{array}$} & INF & 0 & -3.586 & -4.124 & -3.489 & -3.173 \\
\hline & JUROS & 0 & -1.489 & -2.605 & -1.947 & -1.613 \\
\hline & $\triangle J U R O S$ & 0 & -7.206 & -2.606 & -1.947 & -1.613 \\
\hline & DES & 4 & -3.200 & -4.137 & -3.495 & -3.177 \\
\hline & PIB & 0 & -3.883 & -4.124 & -3.489 & -3.173 \\
\hline & OP_INF & 9 & -6.179 & -2.616 & -1.948 & -1.612 \\
\hline & OP_JUROS & 2 & -6.575 & -2.609 & -1.947 & -1.613 \\
\hline & OP_DES & 7 & -6.587 & -2.614 & -1.948 & -1.612 \\
\hline & OP_PIB & 1 & -9.390 & -2.608 & -1.947 & -1.613 \\
\hline \multirow{10}{*}{ 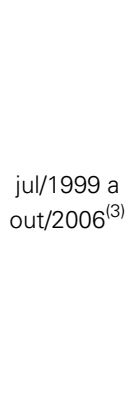 } & INF & 0 & -4.418 & -3.507 & -2.895 & -2.585 \\
\hline & JUROS & 3 & -0.586 & -2.593 & -1.945 & -1.614 \\
\hline & $\triangle J U R O S$ & 2 & -4.848 & -2.593 & -1.945 & -1.614 \\
\hline & DES & 4 & -0.813 & -2.593 & -1.945 & -1.614 \\
\hline & $\Delta \mathrm{DES}$ & 3 & -4.393 & -2.593 & -1.945 & -1.614 \\
\hline & PIB & 0 & -4.405 & -4.067 & -3.462 & -3.157 \\
\hline & OP_INF & 1 & -9.570 & -2.593 & -1.945 & -1.614 \\
\hline & OP_JUROS & 0 & -11.960 & -2.593 & -1.945 & -1.614 \\
\hline & OP_DES & 8 & -6.937 & -2.596 & -1.945 & -1.614 \\
\hline & OP_PIB & 3 & -8.349 & -2.594 & -1.945 & -1.614 \\
\hline
\end{tabular}

Nota: Teste ampliado de Dickey-Fuller (ADF) - o número de defasagens utilizado para cada série foi definido de acordo com o critério de Schwarz.

(1) Para as séries INF, JUROS e DES foi aplicado constante. Para as séries $\triangle D E S, O P \_I N F$, OP_JUROS, OP_DES, OP_PIB não foi utilizado constante ou tendência. Para a série PIB foi aplicado constante e tendência.

(2) Para as séries INF, DES e PIB foi utilizado constante e tendência. Para as demais séries não foi aplicado constante ou tendência.

(3) Para a série INF foi aplicado constante. Para a série PIB foi utilizado constante e tendência. Para as demais séries não foi aplicado constante ou tendência. 